\title{
PARTENARIATO TRA PUBBLICO E PRIVATO NELLA TUTELA E NELLA VALORIZZAZIONE DEI BENI CULTURALI
}

\author{
PIETRO PETRAROIA (*)
}

SuNTO. - Nella normativa italiana sui beni culturali la collaborazione tra enti pubblici e privati è più importante di quanto si creda. La parola "privati" tuttavia ha molti significati; e così la parola "pubblico". Questo saggio racconta l'evoluzione nella normativa sul rapporto pubblico-privato per la valorizzazione dei beni culturali dal 1980 a oggi. Purtroppo oggi mancano ancora normative tecniche e le linee guida, necessarie per favorire chiarezza ed efficacia nella collaborazione fra enti pubblici e privati.

$* * *$

ABSTRACT. - In Italian legislation on cultural heritage, collaboration between public and private bodies is more important than it is believed. However, the word "private" has many meanings, as well as the word "public". This essay tells the evolution of the rules on public-private cooperation for the enhancement of cultural assets from 1980 up today. Unfortunately, still there are no technical standards and guidelines to promote clarity and effectiveness in the collaboration between public and private bodies.

Può essere utile - visto l'argomento di questo convegno e per dare un contesto allo sviluppo del tema che mi è stato assegnato - chiedersi perché in Italia la salvaguardia del patrimonio culturale sia intesa come funzione in primo luogo giuridico-amministrativa, benché esperita da funzionari con formazione tecnico-scientifica, e perché, in particolare, si parli di tutela e non, ad esempio, di cura del patrimonio culturale, come avviene in Germania (con l'espressione Denkmalpflege) o di pro-

(*) Università Cattolica del Sacro Cuore, Milano. Scuola di Specializzazione in Beni storico-artistici. Amministratore delegato di Cultura Valore srl, Italia.

E-mail: p.petraroia@culturavalore.com 
tezione (in inglese/francese: protection; in tedesco: Denkmalschutz). Anzi, in Italia la normativa di settore, dalla formazione dello stato unitario in poi, ha progressivamente sempre più riassorbito nell'ordinamento giuridico le nozioni di conservazione e restauro delle "cose di interesse artistico e storico", ovvero - semplificando - di ciò che oggi definiamo patrimonio culturale, sicché ogni intervento finalizzato a dare continuità non soltanto di sussistenza ma anche di corretta fruizione (pubblica o privata) del patrimonio culturale (pubblico o privato) è oggi regolamentato dalla normativa che chiamiamo di tutela.

Di fatto ciò è avvenuto perché in Italia l'approccio alle testimonianze fisiche della memoria storica è stato guidato da uno sviluppo culturale nel quale le "cose" che siano riconosciute quale documento materiale di tale memoria sono state, per dir così, "personificate": vale a dire che, invece di essere concepite come patrimonio di e per comunità di persone e di cittadini, esse stesse sono state come trasfigurate in soggetto giuridico, titolare di personalità e diritti e capaci di una sussistenza ora e nel futuro, sulla base di forze intrinseche al loro stesso esistere e di motivazioni proprie, come fossero ormai addirittura immortali, sebbene definite "cose".

In tal modo, oggi che la Convenzione di Faro² del 2005 è sottoscritta dal Governo ${ }^{3}$ ma non ancora ratificata dal Parlamento, l'apporto privato alla salvaguardia e al godimento di ciò che è riconosciuto significativo per «preservare la memoria della comunità nazionale e del suo

1 Sul tema rinvio al mio contributo presentato al convegno La valorizzazione dell' eredità culturale in Italia, Macerata, 5-6 novembre 2015, nell'ambito della prima sessione dedicata al tema La Convenzione di Faro e la tradizione culturale italiana, ora edito ne: «Il capitale culturale. Studies on the Value of Cultural Heritage», Supplementi, 5, 2016, 17-28; cfr.: DOI: http://dx.doi.org/10.13138/2039-2362/1552

2 Il testo della Convenzione di Faro è disponibile ovviamente on-line; $\mathrm{cfr}$. ad es.: http://www.beniculturali.it/mibac/multimedia/UfficioStudi/documents/136247 7547947_Convenzione_di_Faro.pdf . Per una presentazione della Convenzione si veda anzitutto: Explanatory Report to the Council of Europe Framework Convention on the Value of Cultural Heritage for Society, Council of Europe Treaty Series, n. 199, in: https://rm.coe.int/CoERMPublicCommonSearchServices/DisplayDCTMContent?d ocumentId=09000016800d3814. Recentemente: M.R. Iacono, La Convenzione di Faro e l'impegno di Italia Nostra, in: Italia Nostra, luglio - agosto - settembre 2016, n. 492, 4-6.

L'adesione del governo italiano ha avuto luogo nella persona del professor Lorenzo Ornaghi, allora Ministro dei Beni e delle Attività Culturali, il 27 febbraio 2013. 
territorio» e per «promuovere lo sviluppo della cultura» ${ }^{4}$ rimane confinato alla funzione di mero supporto finanziario allo sviluppo delle politiche pubbliche, con le modalità dell'atto di mecenatismo o di liberalità ovvero della sponsorizzazione, in questo secondo caso sulla base di un contratto $^{\text {; }}$ intendo dire che sfuma nell'irrilevanza proprio il tema più importante, quello cioè di un rapporto attivo dei cittadini (non soltanto finanziario) e delle loro libere organizzazioni con il patrimonio culturale, che nasca da consapevolezza e corresponsabilità civica, sia cioè nutrito dall'effettivo riconoscimento di valore di quelle «testimonianze aventi valore di civiltà» che sono parte dell'eredità culturale della comunità (per usare linguaggio e valori semantici del testo della Convenzione di Faro $^{6}$ ) e del suo territorio.

Lo svilimento, tuttora, dell'importante ruolo propositivo e attivo, che la Costituzione italiana (art. 4, secondo comma ${ }^{7}$ ) nonché il Codice dei Beni culturali e del Paesaggio (di séguito: Codice BCP), artt. 6 e 111, assegnano ai cittadini "privati” per lo sviluppo della cultura e per la valorizzazione dei beni di appartenenza pubblica, è evidente e grave; esso restringe di fatto alla sola competenza istituzionale pubblica le funzioni di tutela e valorizzazione, lasciando intuire la sfiducia di cui è fatto segno spesso ogni soggetto non statale, soprattutto se privato, che volesse concorrere a tali obiettivi. Del resto, il recente dibattito sulla ulteriore riforma del titolo $\mathrm{V}$ della Costituzione, rispetto alla già pasticciata riforma costituzionale del 2001, ha fatto cogliere un diffuso desiderio di rimontante centralismo statalista proprio in materia di tutela e valorizzazione, alimentato dalla debolezza, ormai consolidatasi da decenni, delle dota-

4 Cfr. l'art. 1 del Decreto Legislativo 22 gennaio 2004, n. 42 "Codice dei beni culturali e del paesaggio, ai sensi dell'articolo 10 della legge 6 luglio 2002, n. 137" (in Gazzetta Ufficiale della Repubblica Italiana, di séguito citata come GU, n. 45 del 24 febbraio 2004 - Suppl. Ordinario n. 28), di séguito citato come Codice BCP.

5 I riferimenti essenziali sono: l'art. 120 del Codice BCP, le norme sui contratti pubblici e in particolare quelle che si sono succedute dal 2006 al 2016.

6 «Articolo 2 - Definizioni. Per gli scopi di questa Convenzione, [...] b. una comunità di eredità è costituita da un insieme di persone che attribuisce valore ad aspetti specifici dell'eredità culturale, e che desidera, nel quadro di un'azione pubblica, sostenerli e trasmetterli alle generazioni future».

7 Costituzione della Repubblica Italiana, art. 4, secondo comma: «Ogni cittadino ha il dovere di svolgere, secondo le proprie possibilità e la propria scelta, un'attività o una funzione che concorra al progresso materiale o spirituale della società». 
zioni umane, finanziarie e strumentali delle Soprintendenze, la cui inevitabile lentezza decisionale viene ormai strumentalizzata per giustificare numerosi atti normativi, volti a limitare su più fronti, all'insegna di una supposta "semplificazione", i loro tradizionali poteri di autorizzazione e controllo. Ma su questo si tornerà più avanti.

Occorre dunque sottrarsi per quanto possibile ad approcci ideologici e strumentali, evitando preconcetti e guardando ai fatti, altrimenti nessuna costruttiva proposta è possibile.

In primo luogo occorre capirsi meglio nel lessico, quando si parla sommariamente di "privati": troppo numerose e variegate sono le soggettività alle quali questa espressione fa sommario riferimento. Ad esempio:

- persone fisiche

- persone giuridiche private con o senza finalità di lucro (incluse le università private e gli enti religiosi di qualsiasi confessione)

- operatori economici a capitale e risorse prevalentemente privati

- organizzazioni non lucrative di utilità sociale (onlus)

- fondazioni (escluse quelle costituite con legge o con fondi prevalentemente pubblici):

> "ordinarie" (ad es. familiari, ai sensi dell'art. 16 del Codice Civile)

> "di partecipazione"

> "di origine bancaria"

- associazioni (con o senza la qualifica di onlus)

Intendiamoci: anche quando si parla di «pubblico» ci si riferisce spesso a soggettività molto diversificate; ad esempio:

- istituzioni ed enti definiti ex art. 114 Cost. (enti locali, territoriali, ed enti afferenti, ad esempio l'istituzione)

- enti con personalità giuridica pubblica, incluse ad es.:

$>$ le autonomie funzionali (ad es.: Camere di Commercio)

> le fondazioni costituite per legge (non quelle ex bancarie)

- università statali

- aziende sanitarie locali

- operatori economici a capitale e risorse prevalentemente pubblici

$>$ enti pubblici non economici

> enti pubblici economici (ad es. azienda speciale)

Nello specifico settore dei beni culturali una serie di provvedimenti normativi volti ad attivare una positiva predisposizione al partenariato genericamente definito "pubblico-privato" si sono succeduti con vicende alterne a partire dal 1982, ossia dalla legge n. 512 di quel- 
l'anno, promossa dall'allora ministro per i Beni culturali e ambientali On.le Vincenzo Scotti, ad otto anni dall'istituzione del ministero stesso ${ }^{8}$ : si trattava del primo tentativo di considerare dazioni e donazioni a favore del patrimonio culturale come atti meritori, dunque degni di "premio" con agevolazioni fiscali di vario tipo, in particolare attraverso una modifica al Testo Unico delle Imposte Dirette. Un tratto particolarmente innovativo era riconoscibile nella previsione di benefici fiscali a privati sia a fronte di un vantaggio per il patrimonio pubblico (ad esempio cessione allo Stato di beni culturali a scomputo di imposte dirette e di tasse di successione, cfr. gli artt. 6 e 7) sia nel caso in cui il vantaggio riguardasse beni di proprietà privata appartenenti al beneficiario stesso (art. 3), così da incentivare l'adozione solerte, da parte dei proprietari, di misure di sicurezza e conservazione a vantaggio di propri beni, i quali, in quanto notificati per il loro valore culturale, sono soggetti a tutela in funzione dell'interesse pubblico.

Questa norma fu salutata alla sua promulgazione da generale consenso, ma venne poi in vario modo depotenziata e limitata quindi nei suoi effetti, per il timore - che reputo del tutto infondato e che comunque non è stato mai sostenuto da prove - che generasse un significativo mancato introito all'erario.

Non ripercorro questa storia ingloriosa per i nostri poteri pubblici: l'esito è stato frequentemente quello di indurre i consulenti fiscali di privati ed imprese a non consigliare ai propri assistiti l'erogazione a favore di beni culturali, preferendo semmai suggerire loro di dichiarare le eventuali spese di sponsorizzazione culturale come oneri sostenuti per pubblicità, accedendo ai relativi benefici fiscali, ovviamente ritenuti più convenienti, a parità di importi deducibili, in quanto non bisognosi di ulteriore certificazione da parte delle competenti Soprintendenze.

Dal 2004 il Codice dei Beni culturali e del Paesaggio e successive modifiche, nella sezione dedicata alla valorizzazione e precisamente all'art. 120, ha riconosciuto piena dignità alla sponsorizzazione per il patrimonio culturale e anzi opportunamente ha incluso in modo estensivo fra gli interventi meritevoli di corrispettivi vantaggi dello sponsor (da precisare con contratto) «ogni contributo, anche in beni o servizi, erogato per la progettazione o l'attuazione di iniziative in ordine alla tute-

8 Legge 2 agosto 1982, n. 512 "Regime fiscale dei beni di rilevante interesse culturale” (in GU n. 216 del 7 agosto 1982). 
la ovvero alla valorizzazione del patrimonio culturale, con lo scopo di promuovere il nome, il marchio, l'immagine, l'attività o il prodotto dell'attività del soggetto erogante [...incluse] iniziative di soggetti privati su beni culturali di loro proprietà».

Peccato che le norme sui contratti pubblici, particolarmente quelle in vigore fra il 2006 e il 2016, abbiano equiparato le sponsorizzazioni per beni culturali pubblici a meri atti di accrescimento dei fondi per lavori pubblici, assoggettandoli perciò alla loro disciplina, con ciò snaturando gravemente quel rapporto di particolare predilezione che connota in genere la scelta da parte dello sponsor del bene culturale o dell'intervento cui destinare proprie risorse, sia pure a fronte di un corrispondente vantaggio promozionale: questo esito, che reputo assolutamente svalutante dell'intervento privato (sia pure non a titolo di mera liberalità), si concretizzava ad esempio nella prescrizione rigida di individuare lo sponsor mediante bando, al fine di assicurare trasparenza e pariteticità di condizioni fra potenziali "concorrenti". Si tratta a mio avviso di un approccio legislativo che non rispettava il principio di proporzionalità della normazione, in quanto il principio della tutela della concorrenza veniva posto non come regola ma come obiettivo prioritario; ma è anche evidente che quella non potesse essere la strada migliore per favorire un coinvolgimento "fervido" dei cittadini nella cura dell'eredità culturale, in quanto l'esito sarebbe stato quello di allontanare tutti i non vincitori, creando delusione, quando non contenzioso.

Di conseguenza, i provvedimenti sopravvenuti con il DecretoLegge 83/2014 (convertito con modificazioni nella legge 106/2014) e detti inopinatamente "Art-bonus", hanno notevolmente migliorato l'appetibilità per le erogazioni liberali da parte di privati, sia per l'entità del vantaggio fiscale sia per una decisa semplificazione delle procedure; va tuttavia osservato che le erogazioni da parte delle imprese, in conseguenza di tale norma, possono invece avere una significativa incidenza soltanto a fronte di fatturati molto consistenti, dovendosi contenere entro il 5 per mille di essi.

9 "ART-BONUS - Credito di imposta per favorire le erogazioni liberali a sostegno della cultura”: così è il titolo dell'art. 1 del Decreto-Legge 31 maggio 2014, n. 83 "Disposizioni urgenti per la tutela del patrimonio culturale, lo sviluppo della cultura e il rilancio del turismo" (in GU n. 125 del 31 maggio 2014) convertito con modificazioni dalla L. 29 luglio 2014, n. 106 (in G.U. n. 175 del 30 luglio 2014). 
Tra i "privati" vanno considerate anche le fondazioni di origine bancaria, alle quali il Codice BCP dedica l'art. 121, prevedendo che il «Ministero, le regioni e gli altri enti pubblici territoriali, ciascuno nel proprio ambito, possono stipulare, anche congiuntamente, protocolli di intesa con le fondazioni conferenti».

Tutto ciò considerato, rimane tuttavia evidente che l'apporto "privato" fatica molto a tradursi in vero partenariato con enti pubblici, perché resta comunque per lo più confinato a quello "esterno" di mero fornitore di beni e servizi alla pubblica amministrazione, ovvero di fonte di risorse a vario titolo (sponsor, mecenate, fondazione ex bancaria...), frequentemente fuori da azioni di programmazione per interventi di impatto territoriale; sostanzialmente inattivata resta infatti quella forma di coinvolgimento maggiormente proattivo e più paritario, che invece è evocata proprio nel Codice BCP all'art. 111, laddove affiora la possibilità per enti pubblici e soggetti privati di convergere corresponsabilmente verso la coproduzione di numerose e differenti azioni di valorizzazione, quali ad esempio la «costituzione ed organizzazione stabile di risorse, strutture o reti, ovvero» la «messa a disposizione di competenze tecniche o risorse finanziarie o strumentali»: azioni, queste, enunciate certo in termini generali, ma tali da suggerire un più vero partenariato strutturato, non occasionale soltanto, tra soggetti pubblici e privati in fase sia di progettazione che di attuazione, con la possibilità di interventi legislativi a livello regionale. La norma arriva a indicare, pur restando finora purtroppo senza concrete conseguenze, che «la valorizzazione ad iniziativa privata è attività socialmente utile e ne è riconosciuta la finalità di solidarietà sociale»: espressione che induce legittimamente ad attendersi un futuro completamento in altra sede normati$\mathrm{va}$, ad esempio in analogia a quanto in via ordinaria è previsto a favore delle organizzazioni non lucrative di utilità sociale (onlus) ${ }^{10}$.

Un avanzamento nella definizione di presupposti normativi più aperti all'idea di partenariato, particolarmente per beni di interesse pubblico e con connotati storico-culturali, si registra nel 2016 con il

10 Sul tema e le discipline connesse: P. Petraroia, Storia (storie?) dell'arte (delle arti?) e valorizzazione, Il capitale culturale, I (2010), 143-148; P. Petraroia, La valorizzazione come dimensione relazionale della tutela, in: AA. VV. (a cura di G. Negri Clementi e S. Stabile), Il diritto dell'arte. 3. La protezione del patrimonio artistico, Milano, Skira, 2014, 41-49. 
d.lgs. $50^{11}$, che innova la regolamentazione sui contratti pubblici. Qui le procedure per l'individuazione di sponsor risultano finalmente un po' meno rigide e scoraggianti.

Mi riferisco anzitutto all'art. 151, che, fin dal titolo, alla fattispecie della sponsorizzazione aggiunge quella delle «forme speciali di partenariato» ${ }^{12}$ : già questa è novità non di poco conto, in quanto l'espressione

11 Decreto Legislativo 18 aprile 2016, n. 50 "Attuazione delle direttive 2014/23/UE, 2014/24/UE e 2014/25/UE sull'aggiudicazione dei contratti di concessione, sugli appalti pubblici e sulle procedure d'appalto degli enti erogatori nei settori dell'acqua, dell'energia, dei trasporti e dei servizi postali, nonché per il riordino della disciplina vigente in materia di contratti pubblici relativi a lavori, servizi e forniture" (in GU n. 91 del 19 aprile 2016 - Suppl. Ordinario n. 10) e successive modificazioni e integrazioni.

12 Nella versione (ora non più in vigore) derivante dalle modifiche introdotte dal terzo correttivo del "Codice dei contratti pubblici relativi a lavori, servizi e forniture", di cui al d.lgs. 12 aprile 2006, n. 163, emanato in attuazione delle direttive 2004/17/CE e 2004/18/CE, veniva data (art. 3, comma 15 ter) la seguente definizione di «contratti di partenariato pubblico-privato»: «contratti aventi per oggetto una o più prestazioni quali la progettazione, la costruzione, la gestione o la manutenzione di un'opera pubblica o di pubblica utilità, oppure la fornitura di un servizio, compreso in ogni caso il finanziamento totale o parziale a carico di privati, anche in forme diverse, di tali prestazioni, con allocazione dei rischi ai sensi delle prescrizioni e degli indirizzi comunitari vigenti». Nel d.lgs. 18 aprile 2016, n. 50, cit. alla prec. nota 11 ed ora in vigore, viene data la seguente definizione di "contratto di partenariato pubblico-privato": «il contratto a titolo oneroso stipulato per iscritto con il quale una o più stazioni appaltanti conferiscono a uno o più operatori economici per un periodo determinato in funzione della durata dell'ammortamento dell'investimento o delle modalità di finanziamento fissate, un complesso di attività consistenti nella realizzazione, trasformazione, manutenzione e gestione operativa di un'opera in cambio della sua disponibilità, o del suo sfruttamento economico, o della fornitura di un servizio connesso all'utilizzo dell'opera stessa, con assunzione di rischio secondo modalità individuate nel contratto, da parte dell'operatore». Più sinteticamente si può fare ricorso alla seguente definizione: «forme di cooperazione tra i poteri pubblici e i privati allo scopo di finanziare, costruire e gestire infrastrutture o fornire servizi di interesse pubblico»; per tale definizione ed un'ampia disamina del tema rinvio a: V. Sessa, Il partenariato pubblico-privato, in: AA. VV., Il nuovo diritto dei contratti pubblici. Commento organico al d.lgs. 18 aprile 2016, n. 50, a cura di F. Caringella, P. Mantini, M. Giustiniani, Roma, Dike giuridica, 2016, 457-491. Dall'ampia bibliografia ivi presentata dall'autrice estraggo alcune citazioni in particolare, relative al periodo tra l'entrata in vigore del d.lgs. 12 aprile 2006, n. 163 sopra citato, e l'emanazione del d.lgs. 50/2016: E. Iossa - F. Russo, Il Partenariato Pubblico-privato in Italia, in Riv. pol. econ., 2008; M.P. Chiti (a cura di), Il Partenariato Pubblico-Privato, Napoli, 2009; F. Caringella - G.F. Cartei - C. Ibba - G. Pericu - A. Petretto - G. Cerrina Feroni (a cura di), Il partenariato pubblico-privato, Torino, 2011; 
"partenariato", specialmente per l'ambito tematico che qui ci interessa, di fatto invita ad un raccordo (sia pure non esplicitato) con il menzionato art. 111 del Codice BCP ed ai suoi aspetti pre-giuridici cui si è fatto cenno.

È vero che l'art. 151 rinvia al precedente art. 19, nel quale il concetto di partenariato non appare; ma esso intanto si applica solo a importi o valori superiori a quarantamila euro, liberando già con questa soglia significative potenzialità specialmente nel settore dei beni culturali mobili. Inoltre, proprio per gli importi che siano superiori a quarantamila euro, non va trascurato che l'art. 19 legittima pienamente la titolarità di un qualsiasi soggetto a proporsi come sponsor di un intervento determinato, che evidentemente la pubblica amministrazione potenzialmente beneficiaria ritiene comunque di poter accettare; in questo caso, la norma prevede semplicemente che la stazione appaltan-

M. Cafagno - A. Botto - G. Fidone - G. Bottino (a cura di), Negoziazioni pubbliche - Scritti su concessioni e partenariati pubblico-privati, Milano, 2013; G.F. Cartei - M. Ricchi (a cura di), Finanza di progetto e partenariato pubblico-privato, Napoli, 2015. Nel suo saggio V. Sessa invita a «guardare al partenariato in termini non riduttivi, valorizzandone non solo l'aspetto di modello concertativo tra enti pubblici ed organizzazioni rappresentative delle parti sociali e delle imprese, ma anche quello di opportunità di condivisione operativa di obiettivi d'interesse comune, capace di potenziare tanto il perseguimento dell'interesse pubblico da parte dell'amministrazione, da una parte, quanto degli interessi particolari del privato, dall'altra. Il partenariato, peraltro,» aggiunge, «costituisce una forma più avanzata della semplice concertazione, nella quale il fine perseguito con intese e accordi è l'interesse generale, ma i soggetti sono spesso attori istituzionali che restano ciascuno espressione di distinti interessi; nel partenariato, infatti, i soggetti tendono ad essere "a fisonomia variabile", ovvero ad integrare fortemente le proprie azioni, facendo convergere istanze individuali verso interessi diffusi allo scopo di sostenere progetti di sviluppo territoriale nei quali la sola concertazione non assicurerebbe, oltre il consenso e un impegno ad assumere responsabilità specifiche da parte di ciascun soggetto, anche un'effettiva interazione. Il ricorso a forme contrattuali innovative acquisisce dunque il suo giusto ruolo se non ci si limita a considerarlo quale modalità alternativa per finanziare interventi e servizi di interesse pubblico bensì, al contrario, quale possibilità di collaborazione tra soggetti che, attraverso la partecipazione al procedimento decisionale relativo alla cosa pubblica, possono recare apporti innovativi e operativi ai processi e progetti di sviluppo locale, in grado in primo luogo di migliorare la qualità dell'azione amministrativa e, in secondo luogo, di risolvere il conflitto tra istanze dei singoli investitori e interessi diffusi della comunità, così superando anche quella distanza troppo spesso percepita tra la società civile e il mondo produttivo, da una parte, e l'amministrazione e la politica di cui essa è espressione, dall'altra». 
te pubblichi sul proprio sito internet un «apposito avviso» con il quale «si comunica l'avvenuto ricevimento di una proposta di sponsorizzazione, indicando sinteticamente il contenuto del contratto proposto. Trascorso il periodo di pubblicazione dell'avviso, il contratto può essere liberamente negoziato, purché nel rispetto dei principi di imparzialità e di parità di trattamento fra gli operatori che abbiano manifestato interesse, fermo restando il rispetto dell'articolo 80», riguardante i motivi di esclusione dagli appalti in generale.

L'art. 19 contiene fra l'altro importanti prescrizioni, di grande opportunità per interventi su manufatti di interesse storico e artistico. La prima riguarda il caso in cui lo sponsor effettui in proprio l'intervento, agendo cioè «direttamente a sua cura e spese». In tal caso, andrà verificato, a cura della stazione appaltante, «il possesso dei requisiti degli esecutori, nel rispetto dei principi e dei limiti europei in materia», fatto di particolare importanza nel caso di interventi di restauro, se solo si considera quanto dispone l'art. 29, comma 6 del Codice BCP: «Fermo quanto disposto dalla normativa in materia di progettazione ed esecuzione di opere su beni architettonici, gli interventi di manutenzione e restauro su beni culturali mobili e superfici decorate di beni architettonici sono eseguiti in via esclusiva da coloro che sono restauratori di beni culturali ai sensi della normativa in materia».

L'altra disposizione dell'art. 19 che mi sembra di particolare significato prevede che, nel caso di sponsorizzazioni per interventi di valore anche superiore a quarantamila euro, «non trovano applicazione le disposizioni nazionali e regionali in materia di contratti pubblici di lavori, servizi e forniture, ad eccezione di quelle sulla qualificazione dei progettisti e degli esecutori»: anche qui, dunque, si riconosce quella specialità della sponsorizzazione rispetto alle forme di finanziamento ordinario che la previgente normativa sugli appalti pubblici aveva ignorato anche nel caso di beni culturali.

Tornando ora all'art. 151 del d.lgs. 50/2016, va chiarito che esso, rispetto al citato art. 19, definisce un regime speciale per le sponsorizzazioni attinenti al patrimonio culturale: è posto infatti nel Titolo VI ("Regimi particolari di appalto") e precisamente a conclusione del Capo III ("Appalti nel settore dei Beni culturali").

Anzitutto vi si conferma quanto disposto dall'art. 19 come da applicarsi anche per l'ambito dei beni culturali, il quale riceve però un'esplicita quanto significativa espansione, giacché vengono inclusi fra i beneficiari ammissibili sia gli istituti e luoghi della cultura (musei, 
biblioteche, archivi, aree archeologiche, parchi archeologici, complessi monumentali) sia istituzioni dello spettacolo notoriamente in cronica sofferenza finanziaria: sono ricompresi infatti i «contratti di sponsorizzazione finalizzati al sostegno degli istituti e dei luoghi della cultura, di cui all'articolo 101 del decreto legislativo 22 gennaio 2004, n. 42, e successive modificazioni, recante Codice dei beni culturali e del paesaggio, delle fondazioni lirico-sinfoniche e dei teatri di tradizione».

Questo ampliamento dell'ambito di applicazione delle sponsorizzazioni da singoli oggetti o serie di oggetti verso beni culturali di natura complessa fino alle istituzioni dello spettacolo è evidentemente frutto della consapevolezza di risorse pubbliche inadeguate, più che di una volontà di coinvolgimento della "comunità di eredità", come "cittadinanza attiva" in interventi di salvaguardia e di produzione culturale; di fatto modifica indirettamente, ampliandone l'ambito di applicabilità, l'art. 120 del Codice BCP, sopra citato.

L'art. 151 si conclude però con un'espansione ulteriore: «Per assicurare la fruizione del patrimonio culturale della Nazione e favorire altresi la ricerca scientifica applicata alla tutela, il Ministero dei beni e delle attività culturali e del turismo può attivare forme speciali di partenariato con enti e organismi pubblici e con soggetti privati, dirette a consentire il recupero, il restauro, la manutenzione programmata, la gestione, l'apertura alla pubblica fruizione e la valorizzazione di beni culturali immobili, attraverso procedure semplificate di individuazione del partner privato analoghe o ulteriori rispetto a quelle previste dal comma 1», ossia rispetto a quanto previsto dal commentato art. 19 della medesima d.lgs 50/2016.

Si tratta di un ampliamento, come si diceva, assai positivo e del tutto inedito quanto ad opportunità e ambiti di applicazione del partenariato: un istituto giuridico profondamente rinnovato, dunque, soprattutto nello specifico ambito dei beni culturali, ma che potrebbe nell'immediato patire per l'eccesso di genericità della formulazione, bisognosa di una regolamentazione applicativa, che si auspica possa essere davvero chiara e facilmente comprensibile per i cittadini.

In particolare, appare indispensabile almeno che gli atti attuativi del d.lgs. 50/2016 chiariscano (e non sembra così complesso farlo) il raccordo fra le nuove disposizioni dell'art. 151 e gli artt. 112-115 del Codice BCP, i quali regolano appunto le forme di gestione "indiretta" dei beni culturali (ossia mediante concessione a soggetti privati) con disposizioni che già da tempo meriterebbero migliore e più diffusa comprensione ed applicazione anche presso gli enti locali. Inoltre vor- 
rei qui evidenziare come proprio quest'ultimo comma dell'art. 151, sopra largamente trascritto, meriti una lettura ed un'applicazione coordinata con un passaggio del Decreto-Legge 83/2014, sopra richiamato a proposito dei benefici fiscali detti "Art-bonus". Su suggerimento di Italia Nostra, infatti, il legislatore, in sede di conversione in legge del decreto, ha previsto che il «credito d'imposta spettante ai sensi del comma 1 è altresi riconosciuto qualora le erogazioni liberali in denaro effettuate per interventi di manutenzione, protezione e restauro di beni culturali pubblici siano destinate ai soggetti concessionari o affidatari dei beni oggetto di tali interventi». ${ }^{13}$

Sembra dunque che i presupposti normativi indispensabili alla progressiva definizione di modalità concrete e anche molto variegate di partenariato - e non soltanto di dazione - tali da consentire di coniugare buona amministrazione e buona cultura tecnica e d'impresa per lo sviluppo della cultura e la salvaguardia dell'eredità culturale siano oggi finalmente disponibili nell'ordinamento italiano.

In realtà ancora non ci si può dire sufficientemente soddisfatti per tre ragioni che ritengo molto importanti. La prima: non è ancora sufficientemente diffusa e padroneggiata la normativa degli ultimi dodici anni sulla valorizzazione del patrimonio culturale, che è letta da taluni addirittura con repulsione (per un pregiudizio ideologico-politico assolutamente errato che equipara valorizzazione a mercificazione), dimenticando che proprio la valorizzazione è funzione di rilievo pubblico costituzionale ed ormai ampiamente regolata per legge, sia pure pressoché soltanto statale, avendo purtroppo la generalità delle Regioni finora rinunciato ad attivarsi come legislatore primario in materia. La seconda: i benefici fiscali si applicano a beni culturali di proprietà pubblica ma

13 L'art. 1 della norma, così come in vigore a séguito delle modifiche successivamente intervenute, dispone: «Per le erogazioni liberali in denaro effettuate nei periodi d'imposta successivi a quello in corso al 31 dicembre 2013, per interventi di manutenzione, protezione e restauro di beni culturali pubblici, per il sostegno degli istituti e dei luoghi della cultura di appartenenza pubblica, delle fondazioni lirico-sinfoniche e dei teatri di tradizione e per la realizzazione di nuove strutture, il restauro e il potenziamento di quelle esistenti di enti o istituzioni pubbliche che, senza scopo di lucro, svolgono esclusivamente attività nello spettacolo, non si applicano le disposizioni di cui agli articoli 15, comma 1, lettere b) e i), e 100, comma 2, lettere f) e g), del testo unico delle imposte sui redditi, approvato con decreto del Presidente della Repubblica 22 dicembre 1986, n. 917, e spetta un credito d'imposta, nella misura del 65 per cento delle erogazioni effettuate». 
non a quelli numerosissimi e ovunque diffusi in Italia, pur essi di interesse pubblico, che sono in proprietà privata (e abbiamo visto quanto numerose categorie di privati vi siano) ${ }^{14}$. La terza ragione è però forse la più importante: a fronte di un'ampia normativa giuridica manca una cultura della normativa tecnica e regolamentare - infatti largamente deficitaria - che coordini disposizioni giuridiche diverse (in questa sede ne abbiamo viste alcune), garantendo protocolli operativi di accettabile complessità e che diano comunque certezza del diritto a cittadini, amministratori, professionisti, imprese, funzionari, su numerosi ambiti di competenza, indirizzando la discrezionalità tecnica dei responsabili della tutela non meno che le competenze organizzative e di programmazione degli investitori e degli sponsor.

La carenza di normazione tecnica è macroscopica e inaccettabile nel settore della conservazione e restauro dei beni culturali, nonostante il legislatore ne abbia sottolineato l'importanza sia all'art 29, comma 5, del Codice BCP ( $« I l$ Ministero definisce, anche con il concorso delle regioni e con la collaborazione delle università e degli istituti di ricerca competenti, linee di indirizzo, norme tecniche, criteri e modelli di intervento in materia di conservazione dei beni culturali»), sia in altri passaggi del medesimo Codice, come all'art. 114. Come ho sottolineato in altre occasioni, l'eccessivo margine di discrezionalità lasciato ai funzionari tecnici della tutela rappresenta un punto di debolezza e non di forza della funzione pubblica per il patrimonio culturale, né può essere superato con drastiche semplificazioni dei procedimenti autorizzativi, come quelle che hanno accresciuto inopportunamente le competenze delle conferenze dei servizi a svantaggio del ruolo delle Soprintendenze ${ }^{15}$.

14 Per un approfondimento della riflessione su quest'aspetto del cosiddetto Art-bonus rinvio all'appendice al mio saggio: Carta del rischio: linee guida e normativa recente. Una lettura critica, in: Economia della cultura, anno XXIV, 2014, n. 3-4, 303320; sul punto, cfr. in partic. le 315-317.

15 Segnalo su questo tema tre miei contributi: P. Petraroia - S. Della Torre, Norme e pratiche senza sistema, in: Economia della Cultura, XVIII, 2008, n. 2, 161-172; P. Petraroia, Tutela e valorizzazione, in: M. Montella - P. Dragoni, Musei e valorizzazione dei Beni culturali. Atti della Commissione per la definizione dei livelli minimi di qualità delle attività di valorizzazione, Bologna, CLUEB, 2010, 42-54; P. Petraroia, La tutela del patrimonio culturale fra rischio di indebolimento ed esigenze di efficienza, in: Italia Nostra, n. 492, ottobre-dicembre 2016, 9-12. 
La mancanza di protocolli operativi integrati e l'assenza di norme tecniche e linee guida valide e comprensibili per tutti non consentono di promuovere e sviluppare la reciproca fiducia fra pubblico e privato in molti settori e specialmente in quello delicato dell'identità culturale; le semplificazioni procedurali inutilmente esagerate ${ }^{16}$, specialmente in assenza di quadri d'insieme di riferimento, non fanno d'altra parte l'interesse delle comunità di eredità, ossia della gente, ma presumono di risolvere problemi reali di tutela del paesaggio e dei beni culturali ricorrendo al centralismo decisorio e riattribuendo al vertice politico (il Ministro) funzioni sostanzialmente tecnico-amministrative, con un'impressionante inversione di rotta rispetto all'indirizzo legislativo affermatosi a partire dal d.lgs. 3 febbraio 1993, n. 29 e riconfermato dalla stessa Corte Costituzionale con sentenza n. 81 del 3 maggio 2013, sulla differenziazione fra politica e amministrazione, come statuito dall'art. 97 della Costituzione.

Il giusto punto di equilibrio non è una mediazione fra esigenze solo in apparenza opposte - di rigorosa tutela e di rapidità nelle procedure di valutazione e approvazione di richieste di autorizzazione a interventi sul patrimonio culturale; si tratta di apprendere sul serio a gestire la complessità dei processi di riconoscimento, gestione e coevoluzione del patrimonio culturale salvaguardandone l'identità (che è identità della Nazione, per citare il Codice $\mathrm{BCP}$ ) e con la partecipazione della popolazione (come sempre raccomandato da Hugues De Varine ${ }^{17}$ ) e questo non è possibile né con la supercentralizzazione delle decisioni né con eccessi di discrezionalità tecnica di singoli funzionari, spesso troppo autoreferenziali, perché non si dispone di quadri metodologici di riferimento e di protocolli strutturati di valutazione e di carattere operativo: né certo può ancora assolvere a questo compito la "Carta del restauro" del 1972.

Compito primario dei vertici politico-istituzionali (e delle stesse

16 Cito ad esempio l'evidente pressione che viene ad esercitarsi sulle Soprintendenze con le procedure previste dal nuovo comma 14-quinquies della legge 7 agosto 1990, n. 241, come sostituito dall'art. 1 del d.lgs. n. 127 del 30 giugno 2016 Norme per il riordino della disciplina in materia di conferenza di servizi, in attuazione dell'articolo 2 della legge 7 agosto 2015, n. 124 (in GU n. 162 del 13 luglio 2016).

$17 \mathrm{H}$. De Varine, Le radici del futuro. Il patrimonio culturale al servizio dello sviluppo locale, Bologna, CLUEB, 2005. 
Camere legislative) non può certo essere dunque quello di sostituirsi all'amministrazione, ma di saper garantire la definizione di strategie ed un'organizzazione funzionale e sostenibile per il perseguimento da parte di tutti dell'interesse pubblico. Non può esservi infatti legittimo e proficuo partenariato pubblico-privato fuori da un chiaro orientamento all'interesse generale, così come la nostra Costituzione impone; lo strumento amministrativo in generale più idoneo nell'adozione di misure non autoritative (quelle che maggiormente possono esprimersi in forme di partenariato pubblico-privato) sembra essere quello contrattuale o pattizio, di derivazione civilistica, ma legittimamente e da tempo applicato nella pubblica amministrazione ${ }^{18}$. Non è evidentemente in se stesso un toccasana, ma non c'è dubbio che il ricorso a forme di normativa pattizia, correttamente individuate ed esercitate, evita le pastoie dell'iperlegificazione, favorisce nei territori la pertinenza e la congruità dell'azione amministrativa e può aiutare la trasparenza e l'imparzialità della sua azione, favorendo la coalizione di risorse ed interessi orientati al bene pubblico, specialmente nell'ambito della salvaguardia, promozione, valorizzazione e fruizione pubblica dei beni culturali, come dimostrano numerose esperienze ${ }^{19}$ e come prescrive del resto proprio il Codice BCP nella sezione che definisce i principi generali della valorizzazione, in particolare all'art. 112.

Concludendo, vale dunque la pena, per contribuire all'individuazione di sviluppi concreti, riassumere quali paiono requisiti fondamentali generali per un corretto rapporto pubblico - privato:

- la chiarezza delle regole applicabili alla definizione di accordi in coerenza con i loro oggetti e finalità

- la gestibilità in trasparenza dei corrispettivi

18 Cfr. la Legge 7 agosto 1990, n. 241 Nuove norme in materia di procedimento amministrativo e di diritto di accesso ai documenti amministrativi (in GU n.192 del 18 agosto 1990), art. 1-bis: «La pubblica amministrazione, nell'adozione di atti di natura non autoritativa, agisce secondo le norme di diritto privato salvo che la legge disponga diversamente».

19 P. Petraroia, La cura del patrimonio storico-culturale come leva di sviluppo del territorio. Una nuova frontiera dell'ottava legislatura, in: Confront, 3, 2005, 43-55; P. Petraroia, Il governo, in: C. Barbati - M. Cammelli - G. Sciullo, Il diritto dei beni culturali, Bologna, Il Mulino, 2006, 165-191; M. Cammelli, Programmazione e gestione delle attività di valorizzazione. Forme convenzionate e modelli operativi, in: M. Montella - P. Dragoni, Musei e valorizzazione... cit., 2010, 233-249. 
- strumenti pattizi non equivoci, soprattutto riguardo a mezzi, fini, ruoli, risorse, strumenti, metodi, controlli, rendicontazione

- il rispetto dei principi di pubblicità e pari opportunità come presupposto per il rispetto della normativa sulla concorrenza

Tutto questo è garanzia di legalità e affidabilità reciproca dei soggetti pubblici e per:

- fornitori di beni, servizi, lavori (privati ed enti pubblici economici)

- mecenati (privati)

- partner (privati e pubblici)

- sponsor (privati)

- proprietari/concessionari/affidatari di beni e servizi culturali, istituti e luoghi della cultura (privati e pubblici)

Non si ceda tuttavia all'illusione che la sola produzione legislativa, quand'anche adeguata alla qualità, complessità e pervasività del nostro patrimonio artistico e storico, possa produrre effetti proficui, durevoli, estesi; ben sappiamo che sono le comunità, a diversa scala, e le loro organizzazioni che determinano, con la loro consapevolezza e responsabilità, le condizioni più idonee al buon esercizio dei poteri pubblici di tutela, così da favorire anche la propositività privata, specialmente nelle azioni di valorizzazione del patrimonio culturale.

Ci sono condizioni socio-culturali che vanno promosse e sostenute per perseguire un simile obiettivo, apparentemente utopistico; la prima è la diffusione di un alto grado di educazione e preparazione culturale, come le statistiche storiche sulla spesa per l'istruzione pubblica in Italia stanno a dimostrare: la capacità delle amministrazioni locali di promuovere e gestire la tutela degli insiemi si è sviluppata in Italia solo ove la tradizione di forte e continuativo impegno per l'istruzione pubblica diffusa si è consolidata per un secolo, in particolare nella Provincia di Bolzano ${ }^{20}$. Importante è poi sviluppare apprezzamento e buone pratiche di cultura della responsabilità sociale dell'impresa, presupposto per un sano riconoscimento di scelte di convenienza reciproca fra sistemi pubblici e privati.

C'è dunque un percorso impegnativo, ma non impossibile, da compiersi ancora sia da parte pubblica che da parte privata per apprendere a cooperare in termini tali, da integrare giusti interessi privati con

20 S. Novello - P. Petraroia, La tutela degli insiemi, in Italia Nostra, n. 481, giugno-luglio 2014, 30-31. 
corretti interessi pubblici, nell'ambito di un ordinamento giuridico sufficientemente integrato e coerente; del resto la nostra compagine sociale è una sola, benché variegata, e non ci è lecito pensare che una buona amministrazione pubblica possa sussistere senza una buona e sana educazione nella dimensione privata. 